\title{
Influence of different levels and sources of resistant starch on faecal quality of dogs of various body sizes
}

\author{
Raphaël Goudez ${ }^{1,2}$, Mickael Weber ${ }^{2}$, Vincent Biourge ${ }^{2}$ and Patrick Nguyen ${ }^{1 *}$ \\ ${ }^{1}$ LUNAM Université, Oniris, Nutrition and Endocrinology Unit, National College of Veterinary Medicine, \\ Food and Science and Engineering, Nantes, F-44307, France \\ ${ }^{2}$ Royal Canin Research Center, Aimargues F-30470, France
}

(Received 28 October 2010 - Revised 1 May 2011 - Accepted 15 May 2011)

\section{Abstract}

In dry-extruded canine diets, starch ileal digestibility varies with the starch source, amount and processing parameters. Starch that escapes duodeno-ileal digestion can affect faecal quality by stimulating colonic bacterial fermentation. The aim of the present study was to assess the effect of various resistant starch (RS) sources and levels on the faecal score of dogs of different breeds and sizes. A total of twenty-one healthy adult female dogs (body weight ranging 5.0-30.6 kg) were used. The maintenance diet for the dogs was supplemented with increasing amounts of RS from two sources: high-amylose starch from maize (to $2.5,4.3$ and $7.4 \%$ RS) and raw potato starch (to 7.4 and $11.4 \%$ RS). Each level of RS was tested over a $7 \mathrm{~d}$ period followed by a $7 \mathrm{~d}$ washout period. Faecal scores were evaluated by one person using a scale ranging from 1 (for hard and dry faeces) to 5 (for liquid stools). Faeces were considered 'optimal' at scores of $2 \cdot 5-3.0$, 'acceptable' at scores of 3.0-3.75 and 'unacceptable' at scores $>3.75$. Small dogs showed very little sensitivity to RS based on the faecal score, while large dogs were quite responsive to RS supplementation. These results suggest that small dogs are poor models for assessing the effect of starch sources on ileal digestibility. They also indicate that a low RS content (strongly affected by source and processing) is an important factor for ensuring an optimal faecal score in large breed dogs.

\section{Key words: Starch: Dog faecal scores}

Faecal characteristics are important parameters for dog breeders and owners to assess diet quality and also digestibility. Various factors such as the source, amount, and quality of starches, proteins, fibres and minerals can affect the digestibility of dry-expanded diets ${ }^{(1)}$. Among these factors, starch digestibility is the most sensitive to extrusion processing. Currently available in vitro methods to assess starch gelatinisation in a complex matrix are unreliable ${ }^{(2)}$. Reports from the field and work in our laboratory have indicated that large breed dogs $(>25 \mathrm{~kg})$ are at a higher risk of exhibiting softer and moister faeces than smaller dogs ${ }^{(3)}$.

Starch represents $20-50 \%$ of dry-extruded diets. Among the nutrients, starch has one of the widest variabilities in terms of digestibility and the flow of undigested material into the colon. Resistant starch (RS) has been defined as 'the sum of starch and starch degradation products not absorbed in the small intestine of healthy individuals ${ }^{\text {,(4). }}$. Starch can be intrinsically resistant depending on its structure. However, starch resistance also can result from configurational changes during the processing or cooling of cooked starch (retrogradation) $^{(4)}$.
The metabolic effects and potential health benefits of RS have been studied extensively in human subjects ${ }^{(5)}$. However, few studies ${ }^{(6,7)}$ have addressed the effects of RS on the canine colonic ecosystem and faecal quality. To our knowledge, no study has assessed the relationship between RS effects and dog characteristics such as breed and size. Accordingly, the aim of the present study was to assess the effects of various amounts of RS on the faecal scores of dogs differing in breed and size.

\section{Materials and methods}

\section{Animals}

A total of twenty-one adult female dogs (6.5 (SD 1.5) years) were used in the present study: six miniature poodles (5.0 (SD 1.0) kg body weight); six miniature schnauzers (6.9 (SD 0.5$) \mathrm{kg}$ body weight); three giant schnauzers $(30.5$ (SD 3.5$) \mathrm{kg}$ body weight); six German shepherds (27.3 (sD 3.3) kg body weight). The study took place in the Nutrition and Endocrinology Unit of the National College of Veterinary Medicine, Food Science and Engineering, Nantes, France

Abbreviations: MS, high-amylose maize starch; PS, potato starch; RS, resistant starch; TS, total starch.

*Corresponding author: Professor P. Nguyen, fax +33 2406877 46, email patrick.nguyen@oniris-nantes.fr 
(Oniris). The dogs were housed individually or in groups (with two to three animals of the same breed) in closed indoor/outdoor enclosures. The experimental protocol adhered to the European Union guidelines and was approved by the Animal Use and Care Advisory Committee of Nantes Veterinary School. The protocol was reviewed and approved by the Royal Canin Ethics Committee.

\section{Basal diet}

The basal diet was a commercial diet intended for dog maintenance, consisting of $43.0 \%$ total starch (TS; from maize meal), $26.7 \%$ protein, $14.9 \%$ fat and $6.9 \%$ total dietary fibre, DM basis (Royal Canin Adult, Aimargues, France). This diet contained $0.6 \%$ RS, assayed as described below. Each dog was fed an amount previously determined to maintain the optimal body weight $(602 \cdot 4$ (SD $25 \cdot 1) \mathrm{kJ} / \mathrm{kg}$ body weight ${ }^{0.75}$ per $\mathrm{d}$ ). Free access to water was available at all times. In the present study, all nutrient concentrations are expressed on a DM basis.

\section{Resistant starch supplementations}

Using different RS supplementations, three studies were performed over a 3-month period. We used two sources of starch to adjust the RS content in tested diets: high-amylose maize starch (MS; Hi-Maize 260; National Starch, Bridgewater, NJ, USA), wherein TS and RS contents were 94.6 and $42.9 \%$ $\mathrm{DM}$, respectively; potato starch flour (PS; Roquette, Lestrem, France), wherein TS and RS contents were 96.9 and $72.8 \%$ $\mathrm{DM}$, respectively. Studies 1 and 2 intended to assess the effects of increasing amounts of RS. In study 1, MS was added to the basal diet so that the RS content (MS-RS, RS from maize starch) was $0 \cdot 6,2 \cdot 5,4.3$ and $7 \cdot 4 \% \mathrm{DM}$ (addition of $0,5,10$ or $20 \%(\mathrm{w} / \mathrm{w}) \mathrm{MS}$, respectively). All dogs were used in this study. In study 2, PS was added to the basal diet in order to increase the RS content (PS-RS) up to $11.4 \%$ DM (addition of $20 \%$ (w/w) PS). Study 3 intended to compare the effects of similar amounts of the two RS. In this study, supplemented diet with PS was only tested on German shepherds. The RS content (PS-TS) was 7.4\% DM. It resulted from $11 \cdot 1 \%(\mathrm{w} / \mathrm{w})$ PS addition to the basal diet. This RS amount was the same as that provided by the highest MS addition in study 1 . Results of both these studies were compared together.

\section{Experimental design}

All diets were tested successively in all dogs over a $7 \mathrm{~d}$ period followed by a $7 \mathrm{~d}$ washout period.

\section{Measurements}

The TS and RS contents in the supplements were measured with the 'TS' and 'RS' enzymatic kits (Megazyme, Wicklow, Ireland). The TS kit measured glucose products following complete hydrolysis of the starch by a thermostable $\alpha$-amylase and amyloglucosidase. The RS kit measured starch digestion by glucose analysis after digestion for $16 \mathrm{~h}$ at $37^{\circ} \mathrm{C}$ in a shaking water-bath with pancreatic $\alpha$-amylase and amyloglucosidase.

For each period, all stools were scored individually using a faecal score ranging from 1 (hard, dry and crumbly stool) to 5 (liquid diarrhoea). Then, they were classified into three categories: 'optimal', defined by a faecal score of 2.5 (well-formed stool, firm and sticky to touch) to 3 (moistformed stool leaving a definite mark on the floor when picked up); 'acceptable', defined by a score of 3-3.75 (most stools have no form and poor consistency); or 'unacceptable', defined by a score $\geq 3 \cdot 75$. No faecal score was $<2 \cdot 5$. All individual faeces produced by dogs of the same breed over a $7 \mathrm{~d}$ period were used to evaluate the frequency of each score range.

\section{Statistical analysis}

Results are presented as the percentage of each score range for each supplementation type and level. Statistical analyses were performed using Statistica 8.0 software (StatSoft, Inc., Tulsa, OK, USA) with the McNemar $\left(\chi^{2}\right)$ test to compare the frequency of the 'optimal' range with and without supplementation. A $P$ value $<0.05$ was considered significant.

\section{Results}

Study 1 (four breeds; basal, 2.5, 4.3 and 7.4\% resistant starch from high-amylose maize starch)

Smaller dogs (miniature poodles and miniature schnauzers) fed the basal diet $(0.6 \%$ RS) presented no unacceptable stools. Faeces were always well formed, with an optimal score frequency of $>90 \%$. In larger dogs, stools with a poor consistency were observed. The frequency of unacceptable scores was 8.5 and $12.1 \%$ in giant schnauzers and German shepherds, respectively.

Regardless of the MS supplementation level (2.5-7.4\% RS), the faecal scores were always optimal in miniature schnauzers (Fig. 1). In miniature poodles, unacceptable scores (6.6\%) appeared with the $4.3 \%$ MS-RS diet. When miniature poodles were fed a $7 \cdot 4 \%$ MS-RS diet, the frequencies of optimal, acceptable and unacceptable scores were $66.0,18.7$ and 15.3\%, respectively.

In giant schnauzers and German shepherds, the frequency of unacceptable soft stools was strongly linked to the level of starch added to the diet. Compared with the unsupplemented diet, the quantity of unacceptable scores was doubled $(P<0.0001)$ with the $2.5 \%$ MS-RS diet $(20.0 \%$ frequency in giant schnauzers and $24 \cdot 1 \%$ frequency in German shepherds). With $4.3 \%$ MS-RS, optimal scores represented $<42.6 \%$ of all scores. Only $15.0 \%$ of faecal scores were optimal among German shepherds fed a diet with $7 \cdot 4 \%$ MS-RS, while $33 \cdot 3 \%$ were unacceptable. Feeding with the $7 \cdot 4 \%$ MS-RS diet was discontinued in giant schnauzers due to very poor faecal quality (i.e. diarrhoea) and the presence of fresh blood and mucus in the stools. 

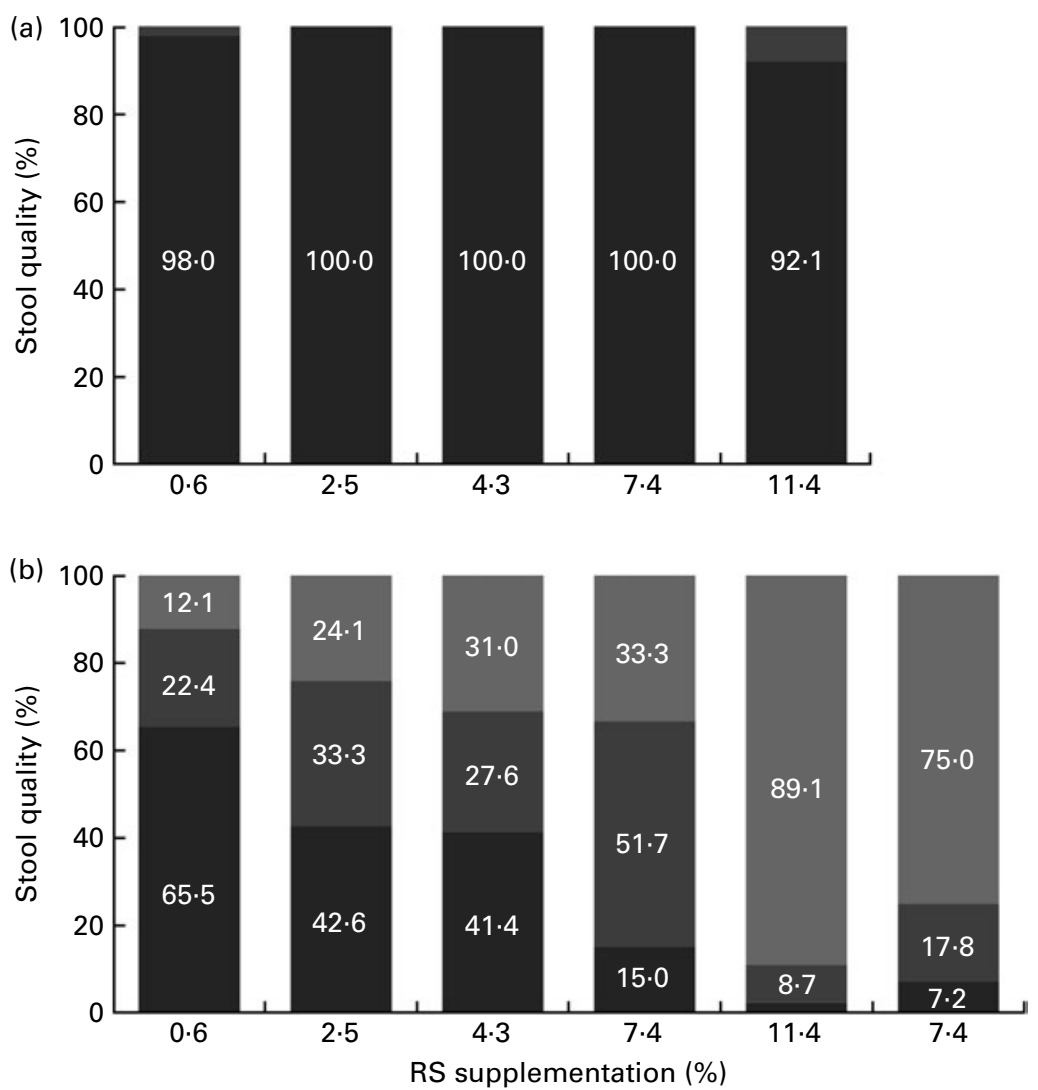

Fig. 1. Influence of different levels of resistant starch (RS) on mean faecal scores in (a) six miniature schnauzers and (b) six German shepherds. Animals were

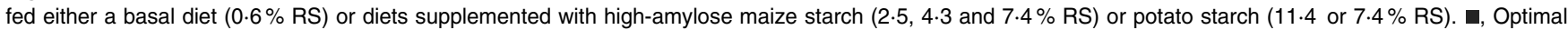
score; $\mathbf{n}$, acceptable score; $\square$, unacceptable score.

\section{Study 2 (three breeds; $11.4 \%$ resistant starch from potato} starch)

When fed the $11.4 \%$ PS-RS diet, the miniature poodles and miniature schnauzers presented well-formed stools with optimal score frequencies of 92.1 and $85 \%$, respectively. In contrast, the frequency of optimal scores among German shepherds was only $2 \cdot 2 \%$, whereas the frequency of unacceptable scores was as high as $89 \cdot 1 \%$.

\section{Study 3 (German shepherds; $7.4 \%$ resistant starch from potato starch)}

When the $7.4 \%$ PS-RS diet was given to German shepherds, the frequency of unacceptable scores was $75.0 \%$. This frequency was only $33.3 \%$ when the same amount of RS was provided by MS supplementation (7.4\% MS-RS in study 1 )

\section{Discussion}

The results of the present study show that dogs respond to indigestible starch differently depending on the size and/or breed. Surprisingly, faecal scores were affected minimally in small breeds (miniature schnauzers and poodles) at all levels of RS supplementation. In contrast, the proportion of unacceptable stools was high even at the lowest level (2.5\% RS) of supplementation in large breed dogs (giant schnauzers and German shepherds). In giant schnauzers fed a diet supplemented with $7.4 \%$ MS-RS, the study had to be discontinued due to signs of colitis (presence of blood and mucus in the faeces), a common complication of excessive carbohydrate fermentation in the colon. This difference in response between small and large dogs was observed with both sources of RS, MS and PS (Fig. 1).

The higher sensitivity of large dogs to RS confirms our previous finding that large dogs fed the same diet at the same energy allowance per kg metabolic body weight present softer and moister faeces than small $\operatorname{dogs}{ }^{(3,8)}$. When the dogs of the present study were fed the commercial unsupplemented diet (study 1), only large dogs presented any unacceptable faecal scores. Large dogs also have been shown to be more sensitive to undigested proteins than small $\operatorname{dog} s^{(1)}$. Although the reason for the higher frequency of lower consistency and moister faeces in large breed dogs remains unclear, it may be explained in part by a longer colonic transit time, higher colonic permeability, proportionally larger colonic volume or higher colonic fermentative activity ${ }^{(9-11)}$. Alternatively, this result could be related to the nature of the undigested residues escaping from the small intestine and their predisposition to ferment. These residues might be able to generate greater biomass and fermentation, leading to higher osmotic pressure and higher water content in the stool. 
While RS supplementation was similar (7.4\% RS) with MS-RS (study 1) or PS-RS (study 3), faecal quality was much lower with PS supplementation. This observation suggests that, as reported previously, PS induces a higher microflora activity than $\mathrm{MS}^{(12)}$. PS is composed of $60 \%$ amylopectin and its enzymatic resistance is conferred by a B-type crystalline form ${ }^{(13,14)}$. The MS used in the present study was composed of $>70 \%$ amylose. The smaller molecule of glucose units has less area for enzymatic activity ${ }^{(15)}$ and its glucose units are more active in hydrogen bonding than amylopectin $^{(16)}$. These differences in starch structure also could be responsible for differences in digestion kinetics or utilisation by the microflora of the dog.

The extrusion processing settings (temperature, steam incorporation, pressure and duration), recipe composition (proportion of starch, protein and fat) and starch source affect the outcome of starch cooking by modulating the molecular changes in granular structure that induce loss of crystallinity $^{(17)}$. As a result, these parameters affect the RS content in the dry-expanded diet ${ }^{(18)}$. Starch processing in complex matrices such as dry-expanded diets is not assessed easily by laboratory methods ${ }^{(1)}$. In vivo tests, although longer and more expensive, are the most appropriate method to determine the response of the dog directly.

Given the relatively high digestive tolerance of miniature schnauzer and poodle dogs despite the high RS concentration used in the present study (11.4\% of measured RS), it appears that small breed dogs would not be good in vivo models to predict starch digestibility and cooking. To our knowledge, the low sensitivity of small dogs to RS has not been reported previously. Further studies are necessary to better understand this phenomenon. In contrast, German shepherd dogs seem to be a good model to discriminate starch cooking and to validate diet processing for dogs in general and for sensitive dogs in particular.

To simulate RS quantities that might be present in commercial diets, supplementations with MS and PS were chosen in the present study, with the final RS supplementation ranging $2 \cdot 5-11.4 \%$, as measured by an RS kit. Nevertheless, we cannot exclude that these RS concentrations could not be representative of starch digestibility in the small intestine of dogs and, therefore, would not be a good predictor of faecal quality.

Indeed, principle of the assay method used in the study was originally developed by Englyst et al. ${ }^{(13)}$ who used a combination of pancreatin, invertase and amyloglucosidase. This method was amended by McCleary et al. ${ }^{(19)}$, who suggested the use of pancreatic amylase and bacterial amyloglucosidase. In addition, the results were correlated with those previously obtained in ileostomised human subjects ${ }^{(20)}$. This verification ensured that the RS content assessed according to McCleary et $a l$. is representative of 'starch resistance' in the human small intestine. We used the same method in the present study, even though the intestinal physiology of dogs is not similar to that of humans (carnivore $v$. omnivore). Therefore, it can be hypothesised that humans and dogs would not digest starch with the same efficiency. The assay method might be unrepresentative of the ileal digestibility in dogs and the measured RS quantities might be different from those actually entering into the colon.

Moreover, the digestive physiology and efficiency vary depending on age ${ }^{(21)}$, dietary habits and, as we have shown, on size or breed ${ }^{(3)}$. Thus, the RS levels might be different between dogs. This possibility could explain the different responses observed in the present study between small and large animals.

In conclusion, the present study confirms our previous findings that large dogs pass softer faeces than small dogs when fed the same diet. Based on our previous work, this observation might be linked in part to the higher colonic fermentative activity of large dogs. Surprisingly, the faecal score of small dogs was quite insensitive to RS supplementation, leading us to question the relevance of RS measurement. We conclude that small dogs appear to be poor models for assessing the effects of processing on starch ileal digestibility. The present results also strongly suggest that low indigestible starch content (affected by source and process) is an important factor for ensuring optimal faecal score in large breed dogs.

\section{Acknowledgements}

We are grateful to Samuel Ninet (National College of Veterinary Medicine, Food Science and Engineering, Nutrition and Endocrinology Unit, Nantes) and his team for their excellent technical assistance. The present study was supported by a grant from Royal Canin SAS, Aimargues, France. R. G. and M. W. designed and supervised the study. P. N. and V. B. provided administrative, technical and material support. R. G. performed the animal experiments and contributed to the analysis of the data and interpretation of the results. R. G. and M. W. wrote the draft of the manuscript. R. G., M. W., V. B. and P. N. critically reviewed the manuscript. V. B. and M. W. were Royal Canin associates.

\section{References}

1. Nery J, Biourge V, Tournier C, et al. (2009) Influence of dietary protein content and source on fecal quality, electrolyte concentrations, and osmolarity, and digestibility in dogs differing in body size. J Anim Sci 88, 159-169.

2. Baks T, Ngene IS, van Soest JJG, et al. (2007) Comparison of methods to determine the degree of gelatinisation for both high and low starch concentrations. Carbohydr Polym 67, 481-490.

3. Weber M, Martin L, Biourge V, et al. (2003) Influence of age and body size on the digestibility of a dry expanded diet in dogs. J Anim Physiol Anim Nutr (Berl) 87, 21-31.

4. Champ M, Langkilde AM, Brouns F, et al. (2003) Advances in dietary fibre characterisation. 2. Consumption, chemistry, physiology and measurement of resistant starch; implications for health and food labelling. Nutr Res Rev 16, 143-161.

5. Champ M, Langkilde AM, Brouns F, et al. (2003) Advances in dietary fibre characterisation. 1. Definition of dietary fibre, physiological relevance, health benefits and analytical aspects. Nutr Res Rev 16, 71-82.

6. Murray SM, Fahey GC Jr, Merchen NR, et al. (1999) Evaluation of selected high-starch flours as ingredients in canine diets. J Anim Sci 77, 2180-2186. 
7. Gajda M, Flickinger EA, Grieshop CM, et al. (2005) Corn hybrid affects in vitro and in vivo measures of nutrient digestibility in dogs. J Anim Sci 83, 160-171.

8. Hernot DC, Biourge VC, Martin LJ, et al. (2005) Relationship between total transit time and faecal quality in adult dogs differing in body size. J Anim Physiol Anim Nutr (Berl) 89, 189-193.

9. Weber MP, Hernot D, Nguyen PG, et al. (2004) Effect of size on electrolyte apparent absorption rates and fermentative activity in dogs. J Anim Physiol Anim Nutr (Berl) 88, $356-365$.

10. Hernot DC, Dumon HJ, Biourge VC, et al. (2006) Evaluation of association between body size and large intestinal transit time in healthy dogs. Am J Vet Res 67, 342-347.

11. Hernot DC, Nery J, Biourge VC, et al. (2008) Colonic permeability is higher in Great Danes compared with smaller breed-dogs. J Anim Physiol Anim Nutr (Berl) 93, 703-709.

12. Bednar GE, Patil AR, Murray SM, et al. (2001) Starch and fiber fractions in selected food and feed ingredients affect their small intestinal digestibility and fermentability and their large bowel fermentability in vitro in a canine model. J Nutr 131, 276-286.

13. Englyst HN, Kingman SM \& Cummings JH (1992) Classification and measurement of nutritionally important starch fractions. Eur J Clin Nutr 46, Suppl. 2, S33-S50.
14. Gallant DJ, Bouchet B, Buleon A, et al. (1992) Physical characteristics of starch granules and susceptibility to enzymatic degradation. Eur J Clin Nutr 46, Suppl. 2, S3-S16.

15. O'Dea K, Nestel PJ \& Antonoff L (1980) Physical factors influencing postprandial glucose and insulin responses to starch. Am J Clin Nutr 33, 760-765.

16. Thorne MJ, Thompson LU \& Jenkins DJ (1983) Factors affecting starch digestibility and the glycemic response with special reference to legumes. Am J Clin Nutr 38, 481-488.

17. Dust JM, Gajda AM, Flickinger EA, et al. (2004) Extrusion conditions affect chemical composition and in vitro digestion of select food ingredients. I Agric Food Chem 52, 2989-2996.

18. Murray SM, Flickinger EA, Patil AR, et al. (2001) In vitro fermentation characteristics of native and processed cereal grains and potato starch using ileal chyme from dogs. J Anim Sci 79, 435-444.

19. McCleary BV, McNally M \& Rossiter P (2002) Measurement of resistant starch by enzymatic digestion in starch and selected plant materials: collaborative study. J AOAC Int $\mathbf{8 5}$, 1103-1111.

20. McCleary BV \& Monaghan DA (2002) Measurement of resistant starch. J AOAC Int 85, 665-675.

21. Meyer H \& Kienzle E (1991) Dietary protein and carbohydrates: relationship to clinical disease. In Purina International Nutrition Symposium, pp. 13-26. Orlando, FL. 